\title{
475518 - CASE REPORT: ANESTHETIC MANAGEMENT FOR MITRAL VALVE REPLACEMENT IN A TRANSPLANTED HEART
}

\section{Ahmed Hegazy, MBBCH, MSc, Fiona Ralley, FRCA, Christopher Harle, MBChB Anesthesia, London Health Sciences Centre, London, ON, Canada}

Introduction: Conventional cardiac surgery after orthotopic heart transplantation is quite rare with an incidence reported to be less than $0.8 \%$ within 15 years of transplantion(1). These cases pose a challenge to both the surgical and anesthesia teams.

Methods: A 55 year old male presented with severe mitral regurgitation 22 years after an orthotopic heart transplant. After a thorough preoperative assessment, the patient was scheduled for an elective mitral valve replacement. His previous heart transplant had been performed secondary to viral myocarditis. He had done very well over the years with regards to his transplant and was maintained on cyclosporine and steroids. Two years ago, he began complaining of exertional chest tightness and shortness of breath. He was diagnosed with graft vasculopathy and underwent percutaneous coronary intervention and stenting of his RCA and LAD. His symptoms had improved until recently he started experiencing increasing shortness of breath and fatigue. Investigations revealed severe mitral regurgitation. In addition, he had developed renal impairment with his long term cyclosporine therapy. The patient had other comorbidities including severe bronchial asthma, pulmonary hypertension and a history of a retroperitoneal bleed post angioplasty. Anesthesia concerns included the risk of hemorrhage with the redo sternotomy, weaning form cardiopulmonary bypass, postbypass coagulopathy, steroid replacement and managing his comorbidities. The surgery proceeded well and a mitral valve replacement with a mechanical valve was performed. Acute normovolemic hemodilution (ANH) was undertaken and the collected blood was fractionated into red blood cells, platelet rich plasma and plasma. These were reinfused after reversal of heparin post cardiopulmonary bypass. Retrograde autologous priming of the cardiopulmonary bypass circuit was performed. Given the recent concerns regarding aprotonin we were unable to use this agent, but tranexamic acid was administered before skin incision and a cell saver device was utilized during the surgery. Coagulation function was monitored by thromboelastography (TEG) in addition to the conventional ACT and INR \& PTT testing. The TEG clearly demonstrated correction of platelet dysfunction following retransfusion of the fractionated blood. Weaning from cardiopulmonary bypass was achieved with titrated infusions of epinephrine, milrinone and vasopressin.

Results: With careful surgical technique and blood conservation strategies, the surgery was concluded without the need for allogenic blood transfusion. The patient was transferred to the cardiac surgery recovery unit in stable condition.

Discussion: Even though valve replacement post-heart transplantion is very uncommon and represents a challenge to both the surgical and anesthesia teams, it is possible to perform such surgery successfully without the need for blood transfusion. We suggest that ANH is an important tool to reduce the need for transfusion of allogenic blood products during cardiac surgery.

References: (1) Heart Surg Forum. 2007 10(2):E110-E114 